\title{
Disappearance of plastic anisotropy in decagonal quasicrystals at small scales and room temperature
}

\author{
Yu Zou ${ }^{1}$, Pawel Kuczera ${ }^{2}$, Walter Steurer ${ }^{2}$ and Ralph Spolenak ${ }^{1, *}$
}

\begin{abstract}
Decagonal quasicrystals (DQCs), as a class of two-dimensional quasicrystals, are known to be highly anisotropic in most of their physical properties. Here we show that their plastic anisotropy can be considerably reduced, and even eliminated, when they are scaled down to the sub-micrometer regime and deformed at room temperature. The reduced plastic anisotropy might be attributed to both size and temperature dependence of dislocation activities. This finding may shed light on the exploration of new deformation mechanisms for quasicrystal plasticity in an unknown size and temperature regime.
\end{abstract}

Keywords: quasicrystals, plastic deformation, anisotropy, size effect, micro-compression 


\section{Introduction}

Quasicrystals are a class of intermetallics that exhibit long-range order but lack translational symmetry [1]. Among them, DQCs consist of quasiperiodic atomic layers with tenfold symmetry but stacked in a periodic order [2, 3]. The distinct atomic arrangements along the tenfold axis (periodic) and within the quasiperiodic layers lead to their strongly direction-dependent properties [4-6], e.g. plastic anisotropy [4]. In metals, plastic anisotropy is attributed to dissimilar dislocation mobilities on different slip systems or preferred crystallographic orientations in polycrystals, i.e. texture. A high level of anisotropy significantly reduces the ductility and formability of a material. For DQCs, the origin of plastic anisotropy is still an interesting topic to be explored for fundamental studies.

Early studies by Feuerbacher et al. $[4,7,8]$ and Edagawa et al. $[9,10]$ unambiguously demonstrated that at high temperatures - above $75 \%$ of their melting temperatures, DQCs exhibit pronounced differences in yield strengths and plastic behavior along different crystallographic directions. For example, in basic cobalt-rich $\mathrm{Al}_{73} \mathrm{Ni}_{10} \mathrm{Co}_{17}[8]$ the yield strength for the loading direction perpendicular to the tenfold axis is about four times higher than for the one inclined at $45^{\circ}$ to the tenfold axis, while the one parallel to the tenfold axis is in between. It is believed that the plastic anisotropy in DQCs is associated with different dislocation characteristics along the quasiperiodic and periodic directions [4].

A different question is whether the plastic anisotropy exists over a wide range of temperature and pressure. There are some hints that the deformation mechanism of quasicrystals would be essentially changed in a low-temperature regime $[11,12]$. Consequently, the plastic anisotropic behavior of the DQCs may become different from that at high temperatures. To explore this question, one could plastically deform DQCs along different orientations at room temperature. This, however, is very difficult, because quasicrystals are extremely brittle at room temperature, failing in a catastrophic way before plastic yielding. Although several attempts have been made to study the room-temperature plasticity in quasicrystals through indentation [13] or under hydrostatic pressures [12], the complex loading modes 
make it difficult to analyze the plastic behavior for each crystallographic direction. Thus, uniaxial compression of quasicrystals at room temperature are still required.

Our prior work showed that an icosahedral quasicrystal, i-Al-Pd-Mn, exhibited an obvious "brittle-toductile" transition in uniaxial compression when the sample size was below around $500 \mathrm{~nm}$ [14]. This result suggests that micro-compression could be an effective way to study the plastic behavior of DQCs at room temperature as well. Therefore, the current study focuses on the plastic deformation of a single crystalline DQC, d-Al-Ni-Co, along three orientations $\left(0^{\circ}, 45^{\circ}, 90^{\circ}\right.$ inclined with respect to the tenfold axis) and with a sample size ranging from $\sim 1 \mu \mathrm{m}$ to $\sim 200 \mathrm{~nm}$ at room temperature.

\section{Materials and Methods}

Single DQCs, d-Al-Ni-Co, were used for this study, because they are highly perfect, stable and intensively studied on their bulk forms. We prepared them from pure metals (Al 99.95\%, Ni 99.99\% and Co $99.99 \%$ ) with an initial composition of $\mathrm{Al}_{77} \mathrm{Ni}_{10.5} \mathrm{Co}_{12.5}$ (Al-excess) and grew them using the self-flux method [15] in an $\mathrm{Al}_{2} \mathrm{O}_{3}$ crucible enclosed in a quartz ampoule under an $\mathrm{Ar}$ atmosphere. The system was heated up to $1200^{\circ} \mathrm{C}$ and slowly cooled down to $1000^{\circ} \mathrm{C}$ at the rate of $1-5 \mathrm{Kh}^{-1}$, resulting in the growth of an agglomerate of several $\sim 0.5 \mathrm{~cm} \times \sim 2-3 \mathrm{~cm}$ decaprismatic crystals. The samples chosen for this study were additionally annealed at $1000^{\circ} \mathrm{C}$ for $48 \mathrm{~h}$ and quenched in water. The X-ray powder diffraction (XRD) and transmission electron microscopy (TEM) diffraction patterns (Fig. 1) of the grown sample indicates a DQC phase, which is comparable to that in literature [2].

Three plate-like samples (2-3 mm thick) were cut using an alumina wheel (Struers 50A13) from the prepared single quasicrystal at angles of $0^{\circ}, 45^{\circ}$, and $90^{\circ}$ with respect to the tenfold axis, polished using 3- $\mu \mathrm{m}$ diamond paste and finally polished using $60-\mathrm{nm} \mathrm{SiO}_{2}$ particle suspension. The actual composition of the DQCs tested in this study is $\mathrm{Al}_{74.4(0.2)} \mathrm{Ni}_{9.3(0.2)} \mathrm{Co}_{16.3(0.1)}$, measured by energy-dispersive $\mathrm{X}$-ray 
spectroscopy $(\mathrm{EDX})$, indicating a basic cobalt-rich phase which is comparable to $\mathrm{Al}_{73} \mathrm{Ni}_{10} \mathrm{Co}_{17}$ in literature [8].

Fine-scale pillars were milled using a focused ion beam (FIB) system (Helios Nanolab 600i, FEI) in two steps: $2.5 \mathrm{nA}$ for coarse milling and 10-40 pA for fine milling. The orientations of the pillars were perpendicular, parallel, and $45^{\circ}$ to the tenfold axis, as indicated by $A_{\perp}, A_{\|}$and $A_{45}$, respectively (Fig. 2a and $2 \mathrm{~b}$ ). For convenience, we adopted the same symbols as those in literature $[4,8]$. The diameters of the FIB-milled pillars are approximately $1 \mu \mathrm{m}, 550 \mathrm{~nm}, 350 \mathrm{~nm}$ and $200 \mathrm{~nm}$ and their aspect ratios are 2.5-4. A taper of $2-3^{\circ}$ was generally observed in those pillars. The diameters of the pillar top were chosen to calculate engineering stresses and evaluate the yield strength at the pillar upper parts. At least four pillars of each size and each orientation were compressed using a nanoindenter (Triboindenter, Hysitron Inc., USA) with a diamond flat-punch tip ( $5 \mu \mathrm{m}$ in diameter, Synton-MDP, Switzerland) in a displacement control mode by feedback mechanism. The strain rate of $2 \times 10^{-3} \mathrm{~s}^{-1}$ was used for all the compression tests. The displacement or loading time was changed according to the pillar height in order to keep the strain rate constant (the detailed procedure is described in [16]). The morphologies of the pillars were characterized using a high-resolution scanning electron microcopy (SEM, FEI Magellan) before and after compression.

\section{Results}

The SEM images show that the pillars in different orientations exhibit rather similar morphologies after compression (Fig. 2c). All three orientations reveal an obvious brittle-to-ductile transition when the pillar size is reduced: the $1-\mu \mathrm{m}$ pillars fail catastrophically during compression without notable plasticity, the 500-nm pillars are plastically deformed with clear shear bands traversing along the samples, and the 200$\mathrm{nm}$ pillars show localized plastic deformation at their upper parts. Although it is hard to identify possible slip planes based on the SEM images, the shear planes are tilted $30-60^{\circ}$ relative to the loading axis for all the orientations and multiple shear lines can be identified. Interestingly, the shear bands shown here are 
not as sharp as those observed in typical fcc metal pillars [17] or semiconductor pillars [18], but exhibit highly wavy, or zigzag-like, morphologies (see high-magnification SEM images in Fig. 3), which are very similar to those in metallic glass pillars [19]. The wavy morphologies of the slip traces could be associated with the wavy morphology might be attributed to extensive cross-slip due to their nonplanar core structure. The localized deformation at the pillar upper part might be attributed to the geometrical taper as well as a strain softening effect, which has been observed in bulk decagonal quasicrystals [7, 8].

Fig. 4 shows the stress-strain curves of the pillars upon compression. We can further confirm that the large pillars $(\sim 1 \mu \mathrm{m})$ have limited ductility—fracture at $\sim 5 \%$ strain, the 500-nm pillars present better ductility than the $1-\mu \mathrm{m}$ pillars, failing at $\sim 10 \%$ strain, while the small pillars $(\sim 200-300 \mathrm{~nm})$ show remarkably ductility - over $30 \%$ strains without fracture. All the pillars exhibit a displacement-burst behavior which has been commonly observed in both metal [17] and metallic glass pillars [19]. The displacement bursts, or force drops, could be associated with the intermittent nucleation and propagations of dislocations in a confined small volume. The first displacement burst can be attributed to the initiation of the dislocsation motion on a specific plane-plastic yielding. Thus, the flow stress at the first displacement burst could be regarded as yield strength. We can observe a slightly increased yield strength as the pillar size decreases for both the $\mathrm{A}_{\perp}$ and $\mathrm{A}_{\|}$orientations, but a relatively larger increase for the $\mathrm{A}_{45}$ orientation.

If we assume that the shear bands are tilted about $45^{\circ}$ relative to the loading direction and result from a pure slip, which means that the Schmid factor is 0.5 , we can obtain the critical resolved shear stress (CRSS) as a function of the pillar diameter, as shown in Fig. 5. When the pillar size is large $(\sim 1 \mu \mathrm{m})$, the $A_{\perp}$ oriented pillars show the highest strength levels, the strength of $A_{\|}$oriented pillars is slightly lower than that of $A_{\perp}$, while the $A_{45}$ oriented pillars show the lowest strength. This order of strength is identical to that reported for bulk compression, as $A_{\perp}>A_{\|}>A_{45}$. As evaluated by the log-log slope, the $A_{\perp}, A_{\|}$and $\mathrm{A}_{45}$ pillars show size-effect exponents of $-0.35,-0.33$ and -0.44 , respectively. At pillar sizes of $\sim 200-300$ 
$\mathrm{nm}$, the CRSS for all the three orientations are converged to the same value of about $3 \mathrm{GPa}$ - the plastic anisotropy has disappeared. The obtained CRSS of the DQCs in our experiments are very close to the simulated shear stress for the pure glide DQCs $(G / 27)$ in the tenfold plane [20] and approach the theoretical strength $(G / 10)$ [21], where $G$ is the shear modulus and about $80 \mathrm{GPa}$ [22]. The observed scattering of strengths could be due to the statistical distribution of defects or dislocation sources in a sample, as demonstrated in pure metal pillars [23].

In a three dimensional (3D) graph of yield strength vs. sample size vs. temperature (Fig. 6), we can compare the plastic anisotropy of bulk quasicrystals in literature [8] with our DQC pillars in a large temperature and size range. By reducing the sample size and the testing temperature, the DQCs change their mechanical behavior from plastic anisotropy (with a factor of 4) to plastic isotropy (a factor of $\sim 1$ ).

\section{Discussion}

Although d-Al-Ni-Co have eight different ordering states, such as $\mathrm{Al}_{70} \mathrm{Ni}_{15} \mathrm{Co}_{15}$ [7], $\mathrm{Al}_{73} \mathrm{Ni}_{10} \mathrm{Co}_{17}$ [8] and $\mathrm{Al}_{70} \mathrm{Ni}_{21} \mathrm{Co}_{9}$ [24], all of them show some degrees of plastic anisotropy at high temperatures, as $\mathrm{A}_{\perp}>\mathrm{A}_{\|}>$ $\mathrm{A}_{45}$. Feuerbacher and Schall [4] summarized distinct dislocation characteristics and correlated with different deformation geometries: in $\mathrm{A}_{45}$, periodic dislocations, i.e. Burgers vectors parallel to the periodic direction; in $\mathrm{A}_{\perp}$, quasiperiodic dislocations, i.e. Burgers vectors within quasiperiodic planes and mixed dislocations i.e. Burgers vectors contain both periodic and quasiperiodic components; in $A_{\|}$, by both periodic and mixed dislocations. The bulk compression demonstrates that the lattice resistance, i.e. Peierls barrier, at high temperatures for periodic dislocations is lower than that for quasiperiodic dislocations. It is also well known that for metal micro-pillars, generally, $\tau_{\mathrm{CRSS}} \sim \tau_{0}+K G b / \lambda+G b \downarrow_{\rho}$ [25], where $\tau_{0}$ is the lattice resistance, $K$ the source-strengthening constant in the order of $0.1, b$ the Burgers vector, $\lambda$ the length of dislocation source, and $\rho$ the dislocation density. Thus, both lattice friction and dislocation interactions can influence the size dependence of the strength in the same material system [26, 27]. 
The result in Fig. 5 indicates that the lattice resistance for the $A_{45}$ orientation is still lower than those for the $A_{\perp}$ and $A_{\|}$orientations, but their strengths are on the same levels when the sample size is scaled down to 200-300 nm. A few mechanisms could account for this reduced plastic anisotropy: first, as temperature decreases, the lattice resistance associated with the motions of periodic and quasiperiodic dislocations may both increase and approach to their extreme values at $0 \mathrm{~K}$, which could be much less anisotropic. Second, as the sample dimension is reduced, its strength becomes more nucleation-controlled rather than propagation-controlled, and the length of the dislocation source (e.g. kinks) can be related to columnar clusters [28], which become much less anisotropic in the nanometer-size regime. Third, the high stress and short dislocation lengths may activate alternative slip systems, leading to multiple slips and reduced anisotropy, which have been observed in sub-micrometer-sized magnesium [29] and high-entropy alloys [30].

Our results demonstrate that the pronounced plastic anisotropy in bulk DQCs in the Al-Ni-Co system can be considerably reduced at small scales and room temperature. However, there is still much to be explored between the regime of high-temperature bulk deformation and that of room-temperature smallscale deformation, as illustrated in Fig. 6. For example, at what size and temperature one should observe a transition from bulk behavior to small pillar behavior is interesting for future studies. It should be noted that dislocation climb has been observed in plastic deformation of d-Al-Ni-Co at high temperatures [31] and of i-Al-Pd-Mn under hydrostatic pressures [12]. At low temperatures and under uniaxial compressions, diffusion is believed to be difficult. How the climb process might contribute to the plastic deformation of small-sized d-Al-Ni-Co at room temperature is still not clear so far and the detailed analysis of dislocation characteristics is still needed. Our studies also call for a detailed modeling of dislocation nucleation and propagation in quasicrystals in a large size and temperature regime.

A final interesting point is that because of the high atomic percentage of aluminum as well as high yield strengths, our quasicrystal micro-pillars offer extremely high specific-yield-strength values (strength-to- 
weight ratios) of about $1 \mathrm{MJkg}^{-1}$, and on this basis, they surpass all the metallic micro-pillars reported to date (Fig. 7). Our study suggests the possibility of using quasicrystals as strong-and-ductile materials. Together with their interesting physical properties [32-34], small-sized quasicrystals may be potentially interesting for engineering applications in small-dimensional devices. 


\section{Acknowledgements}

We thank S. Takeuchi (Tokyo University of Science) and K. Edagawa (The University of Tokyo) for their helpful discussions, A. Sologubenko (LNM, ETH Zurich) for the TEM characterization, ScopeM (ETH Zurich) for supplying electron microcopy facility and A. Evans for proofreading. Y.Z acknowledges the financial support through the Swiss National Science Foundation (SNF Grant: 200021_143633). 


\section{Figure captions}

Fig. 1. (a) Powder diffraction $(\mathrm{Cu}, \mathrm{K} \alpha 1)$, (b) TEM diffraction pattern, and (c) high-resolution TEM image with Fast Fourier Transform (FFT) for the as-prepared d-Al-Ni-Co before deformation, indicating a single decagonal quasicrystal phase.

Fig. 2. Micro-compression of decagonal quasicrystal pillars: (a) the schematic of a decagonal Al-Ni-Co quasicrystal showing quasiperiodic planes (tenfold surfaces) stacked along the periodic direction (tenfold axis). (b) Schematics of micro-pillars with three orientations: perpendicular to tenfold axis $\left(\mathrm{A}_{\perp}\right)$, parallel to tenfold axis $\left(\mathrm{A}_{\|}\right)$and $45^{\circ}$ to tenfold axis $\left(\mathrm{A}_{45}\right)$. (c) Corresponding SEM images of compressed pillars with the diameters of approximately $1 \mu \mathrm{m}, 500 \mathrm{~nm}$ and $200 \mathrm{~nm}$.

Fig. 3. High-magnification SEM images of the deformed pillars ( $500 \mathrm{~nm}$ in diameter) (a) $\mathrm{A}_{\perp}$ and (b) $\mathrm{A}_{45}$, showing wavy morphologies and multiple slips.

Fig. 4. Representative engineering stress-strain curves for (a) $A_{\perp}$, (b) $A_{\|}$and (c) $A_{45}$ oriented pillars with the diameters ranging from $\sim 1 \mu \mathrm{m}$ to $\sim 200 \mathrm{~nm}$. (To identify each curve, the curves are shifted to each other horizontally).

Fig. 5. The relation between critical resolved shear stress (CRSS) and pillar diameters for $A_{\perp}, A_{\|}$, and $A_{45}$ orientations. The size-effect exponents $(m)$ for $\mathrm{A}_{\perp}, \mathrm{A}_{\|}$and $\mathrm{A}_{45}$ are $-0.35,-0.33$ and -0.44 , respectively. The shear stress in the tenfold planes is $G / 27$ by simulation [20] and the theoretical strength is $G / 10$, where $G$ is the shear modulus of about $80 \mathrm{GPa}$ [22]. 
Fig. 6. A 3D illustration showing the size and temperature dependence of yield strengths for the decagonal Al-Ni-Co bulk [8] and the fine-scale pillars in this study. The strength anisotropy is reduced from a factor of $\sim 4$ in bulk forms $(\sim 1.5 \mathrm{~mm})$ at $\sim 800^{\circ} \mathrm{C}$ to identity at small sizes $(\sim 200-300 \mathrm{~nm})$ and room temperature.

Fig. 7. Comparison of the small-sized quasicrystal pillars with other metal and metallic-glass pillars. Ashby map (designed with CES EduPack 2014) of yield strength vs. density, indicating d-Al-Ni-Co quasicrystal pillars exhibit the highest specific strength (the strength-to-weight ratio). (Abbreviations: MG-metallic glass, NL-nanolaminate, NC-nanocrystalline, SC-single crystalline and CScolumnar structured.) Literature data for pillar strengths: pure metals $\mathrm{Au}$ [35], $\mathrm{Al}$ [36], $\mathrm{Ni}$ [37], $\mathrm{Cu}$ [38], Nb, Ta, Mo and W [39], and Mg [40], TiAl [41], NC Cu [42], Ni [43], Ni-W [44], Pt [45] and Rh [46] pillars, NbTaMoW [30, 47], and $\mathrm{Cu}-$ and Zn-based MGs [19]. 


\section{References}

[1] Shechtman D, Blech I, Gratias D, Cahn JW. Phys Rev Lett 1984;53:1951.

[2] Tsai AP, Inoue A, Masumoto T. Mater T Jim 1989;30:150.

[3] Steurer W, Haibach T, Zhang B, Kek S, Lück R. Acta Crystallographica Section B: Structural Science 1993;49:661.

[4] Feuerbacher M, Schall P. Scripta Materialia 2003;49:25.

[5] Park JY, Ogletree DF, Salmeron M, Ribeiro RA, Canfield PC, Jenks CJ, Thiel PA. Science 2005;309:1354.

[6] Freedman B, Lifshitz R, Fleischer JW, Segev M. Nat Mater 2007;6:776.

[7] Feuerbacher M, Bartsch M, Grushko B, Messerschmidt U, Urban K. Philosophical Magazine Letters 1997;76:369.

[8] Schall P, Feuerbacher M, Urban K. Philos Mag 2004;84:705.

[9] Edagawa K, Arai Y, Hashimoto T, Takeuchi S. Materials Transactions, JIM 1998;39:863.

[10] Edagawa K, Ohta S, Takeuchi S, Kabutoya E, Guo J, Tsai A-P. Materials Science and Engineering: A 2000;294:748.

[11] Mukhopadhyay NK, Belger A, Paufler P, Gille P. Philos Mag 2006;86:999.

[12] Mompiou F, Caillard D. Acta Mater 2004;52:3613.

[13] Wollgarten M, Saka H, Inoue A. Philosophical Magazine A 1999;79:2195.

[14] Zou Y, Kuczera P, Sologubenko A, Sumigawa T, Kitamura T, Steurer W, Spolenak R. Superior room-temperature ductility of typically brittle quasicrystals at small sizes (unpublished)

[15] Canfield PC, Fisk Z. Philos Mag B 1992;65:1117.

[16] Zou Y, Spolenak R. Philosophical Magazine Letters 2013;93:431.

[17] Uchic MD, Dimiduk DM, Florando JN, Nix WD. Science 2004;305:986.

[18] Ostlund F, Rzepiejewska-Malyska K, Leifer K, Hale LM, Tang YY, Ballarini R, Gerberich WW, Michler J. Adv Funct Mater 2009;19:2439.

[19] Chen CQ, Pei YT, De Hosson JTM. Acta Mater 2010;58:189.

[20] Mikulla R, Roth J, Trebin H. Philosophical Magazine B 1995;71:981.

[21] Courtney TH. Mechanical behavior of materials: Waveland Press, 2005.

[22] Takeuchi S, Edagawa K. Chapter 8 Elastic and plastic properties of quasicrystals. In: Takeo F, Yasushi I, editors. Handbook of Metal Physics, vol. Volume 3. Elsevier, 2008. p.267.

[23] Bei H, Shim S, Pharr GM, George EP. Acta Mater 2008;56:4762.

[24] Schall P, Feuerbacher M, Urban K. Philosophical Magazine Letters 2004;84:471.

[25] Parthasarathy TA, Rao SI, Dimiduk DM, Uchic MD, Trinkle DR. Scripta Materialia 2007;56:313.

[26] Korte S, Clegg WJ. Philos Mag 2011;91:1150.

[27] Zou Y, Spolenak R. Philos Mag 2015;95:1795.

[28] Cervellino A, Haibach T, Steurer W. Acta Crystallographica Section B: Structural Science 2001;58:8.

[29] Yu Q, Qi L, Mishra RK, Li J, Minor AM. Proceedings of the National Academy of Sciences 2013;110:13289.

[30] Zou Y, Maiti S, Steurer W, Spolenak R. Acta Mater 2014;65:85.

[31] Bartsch M, Schall P, Feuerbacher M, Messerschmidt U. J Mater Res 2005;20:1814.

[32] Dubois J-M. Chemical Society Reviews 2012;41:6760.

[33] Dolinsek J. Chemical Society Reviews 2012;41:6730.

[34] de Boissieu M. Chemical Society Reviews 2012;41:6778.

[35] Greer JR, Oliver WC, Nix WD. Acta Mater 2005;53:1821.

[36] Kunz A, Pathak S, Greer JR. Acta Mater 2011;59:4416.

[37] Frick CP, Clark BG, Orso S, Schneider AS, Arzt E. Materials Science and Engineering: A 2008;489:319.

[38] Jennings AT, Burek MJ, Greer JR. Phys Rev Lett 2010;104:135503. 
[39] Schneider AS, Kaufmann D, Clark BG, Frick CP, Gruber PA, Monig R, Kraft O, Arzt E. Phys Rev Lett 2009;103.

[40] Lilleodden E. Scripta Materialia 2010;62:532.

[41] Edalati K, Toh S, Iwaoka H, Watanabe M, Horita Z, Kashioka D, Kishida K, Inui H. Scripta Materialia 2012;67:814.

[42] Okamoto NL, Kashioka D, Hirato T, Inui H. Int J Plasticity 2014;56:173.

[43] Rinaldi A, Peralta P, Friesen C, Sieradzki K. Acta Mater 2008;56:511.

[44] Jang D, Greer JR. Scripta Materialia 2011;64:77.

[45] Gu XW, Loynachan CN, Wu ZX, Zhang YW, Srolovitz DJ, Greer JR. Nano Lett 2012;12:6385.

[46] Alshehri O, Yavuz M, Tsui T. Acta Mater 2013;61:40.

[47] Zou Y, Ma H, Spolenak R. Nat Commun 2015;6. 


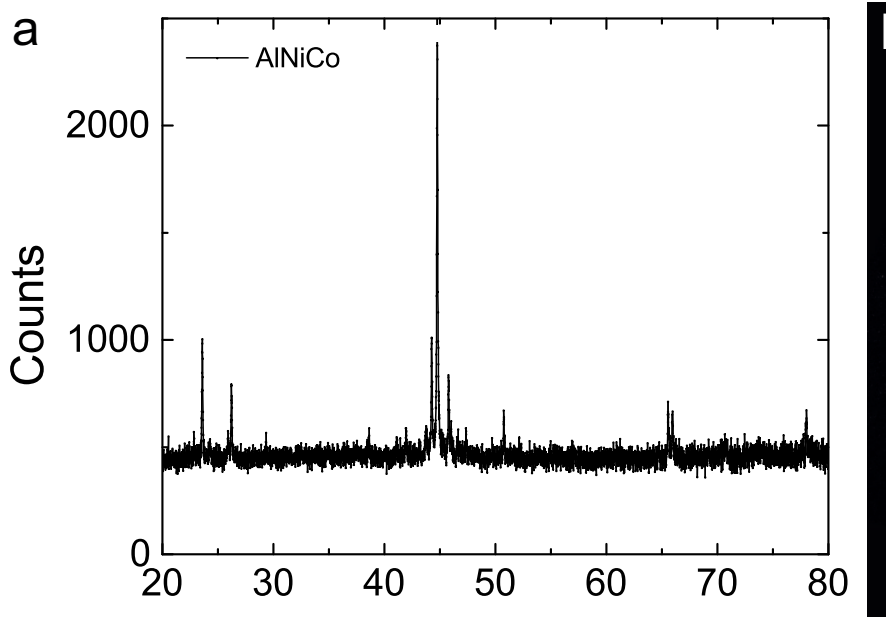

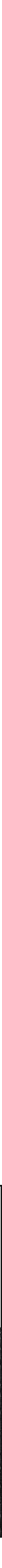

Figure 1
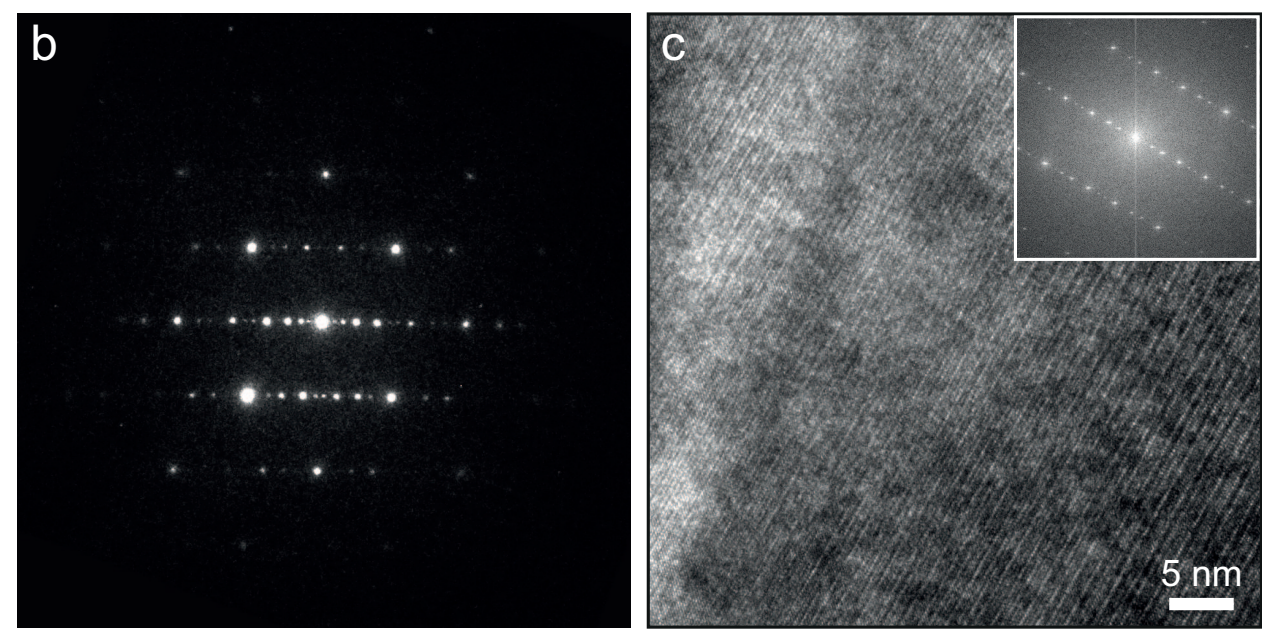

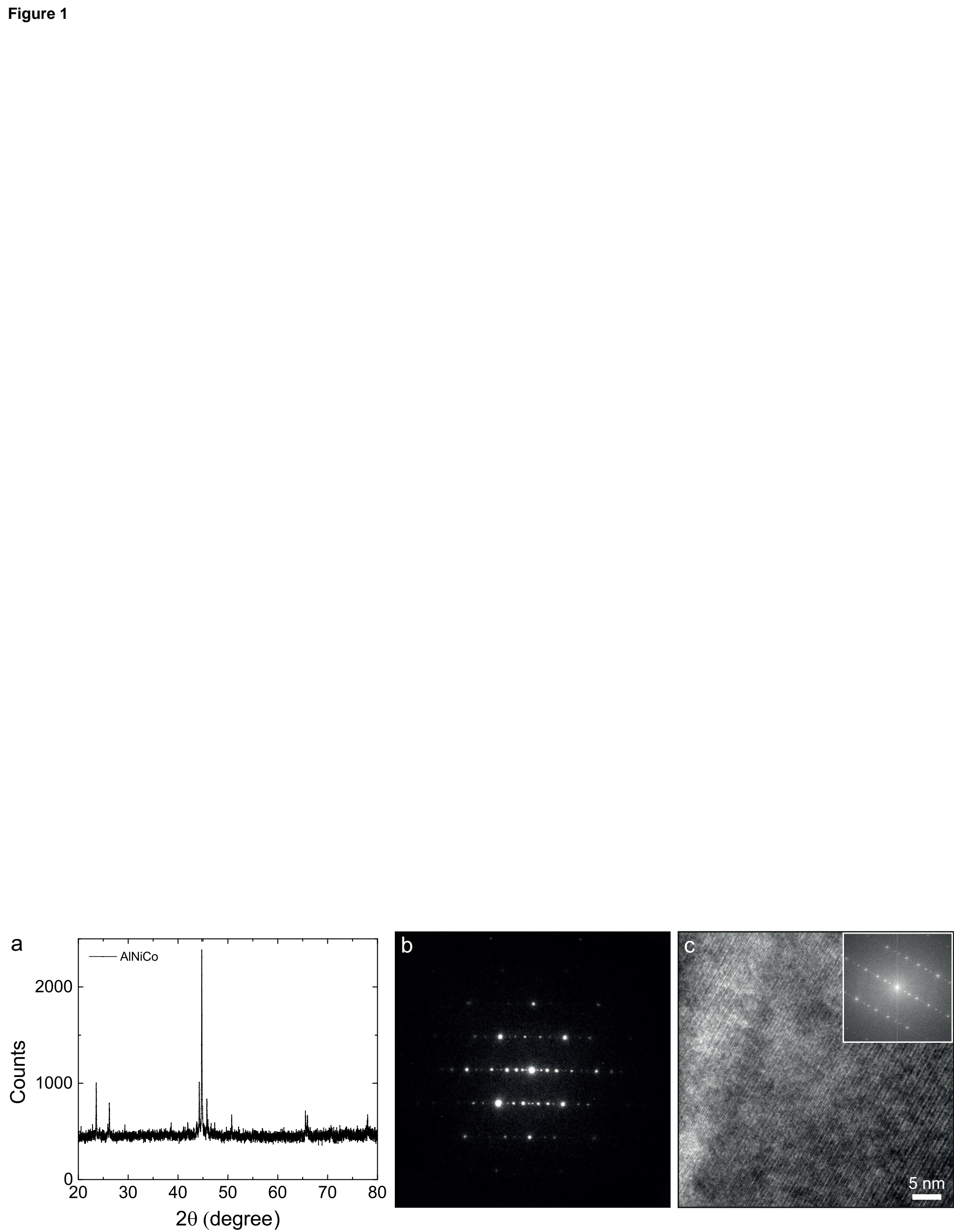



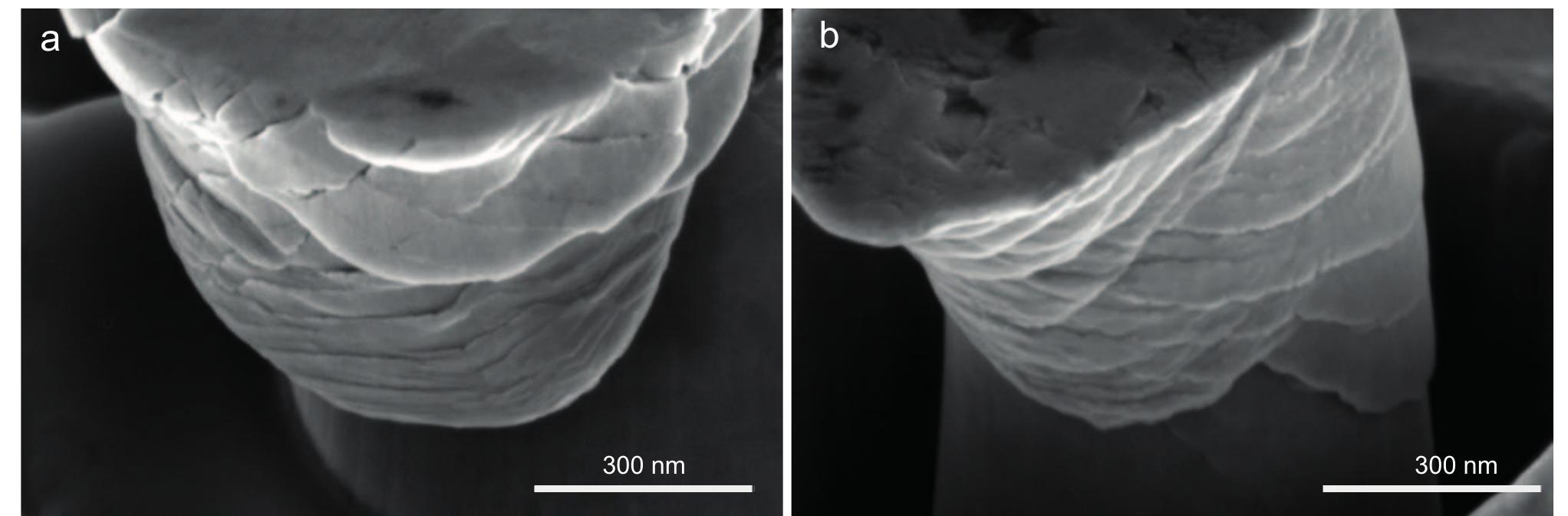

Figure 3

3

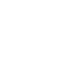

Figure 
Figure 4

a

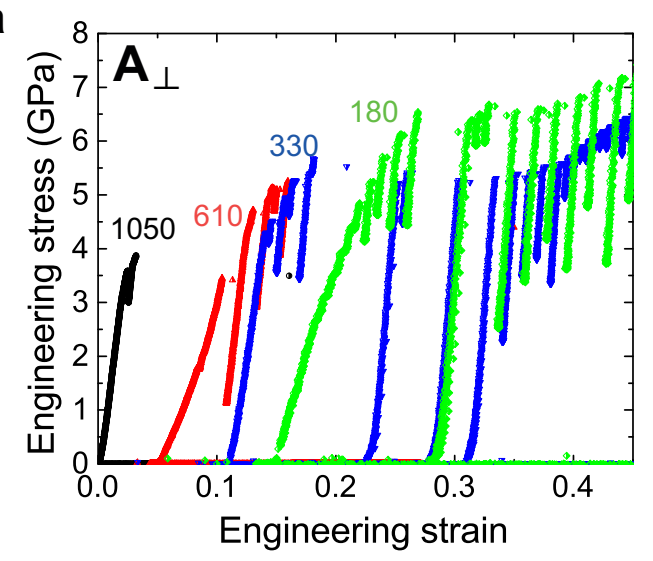

b

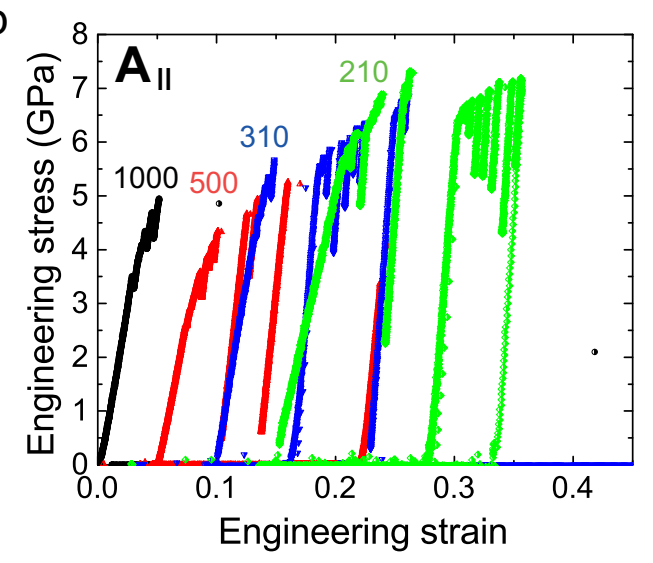

C

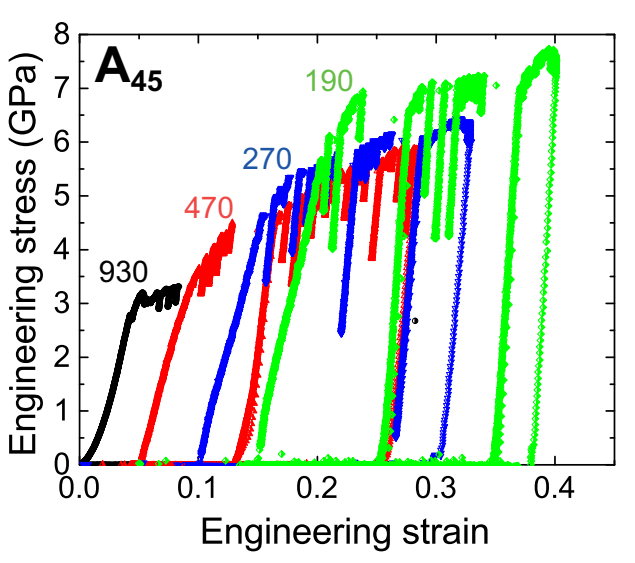




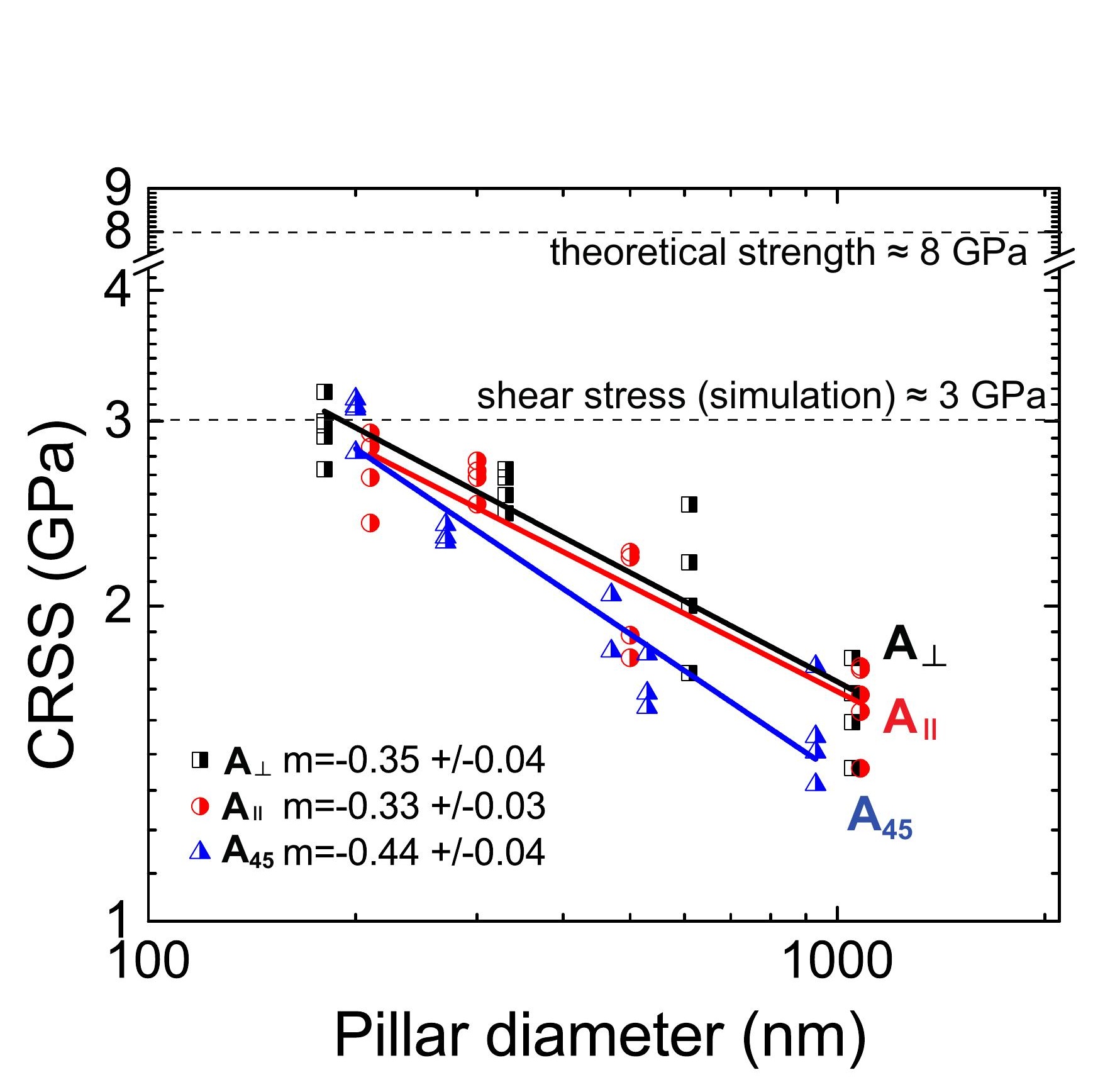

Figure 5

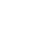

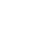
-

(2n)
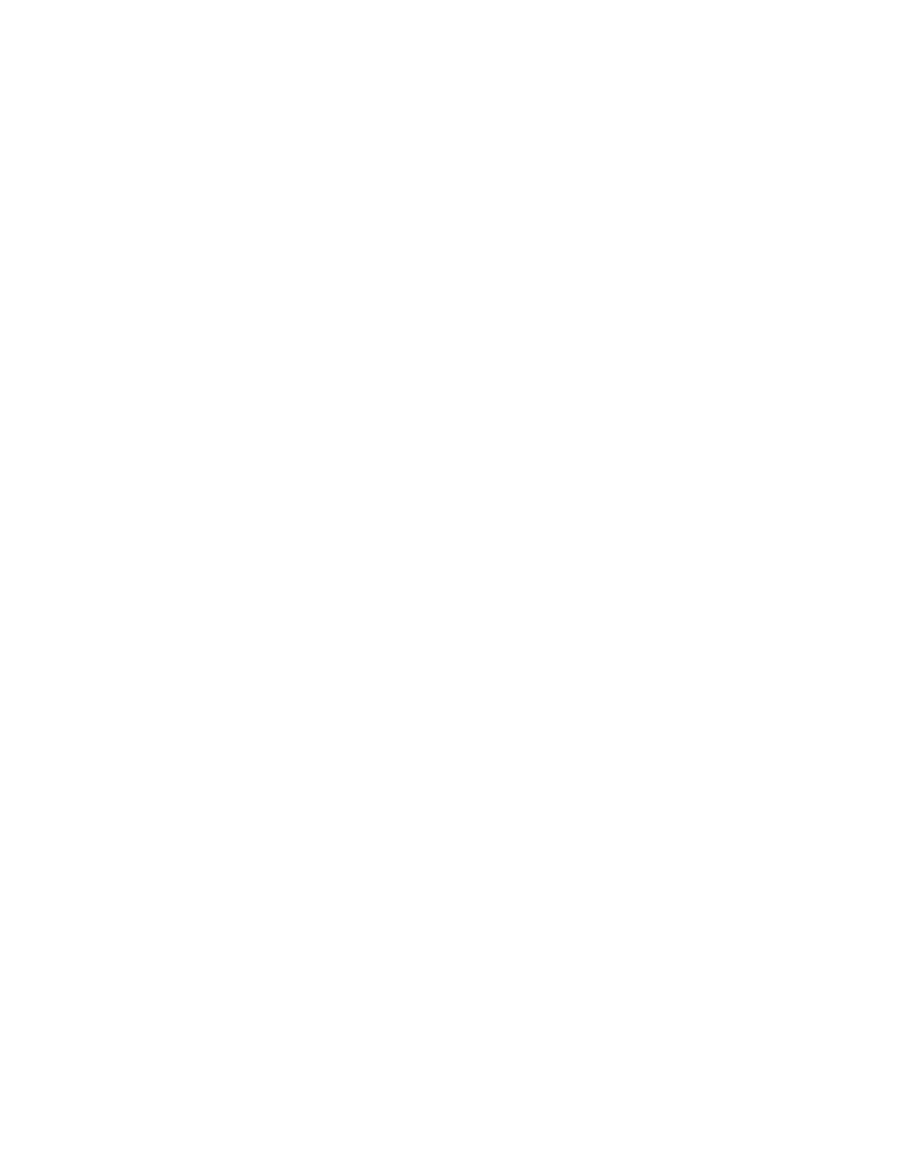


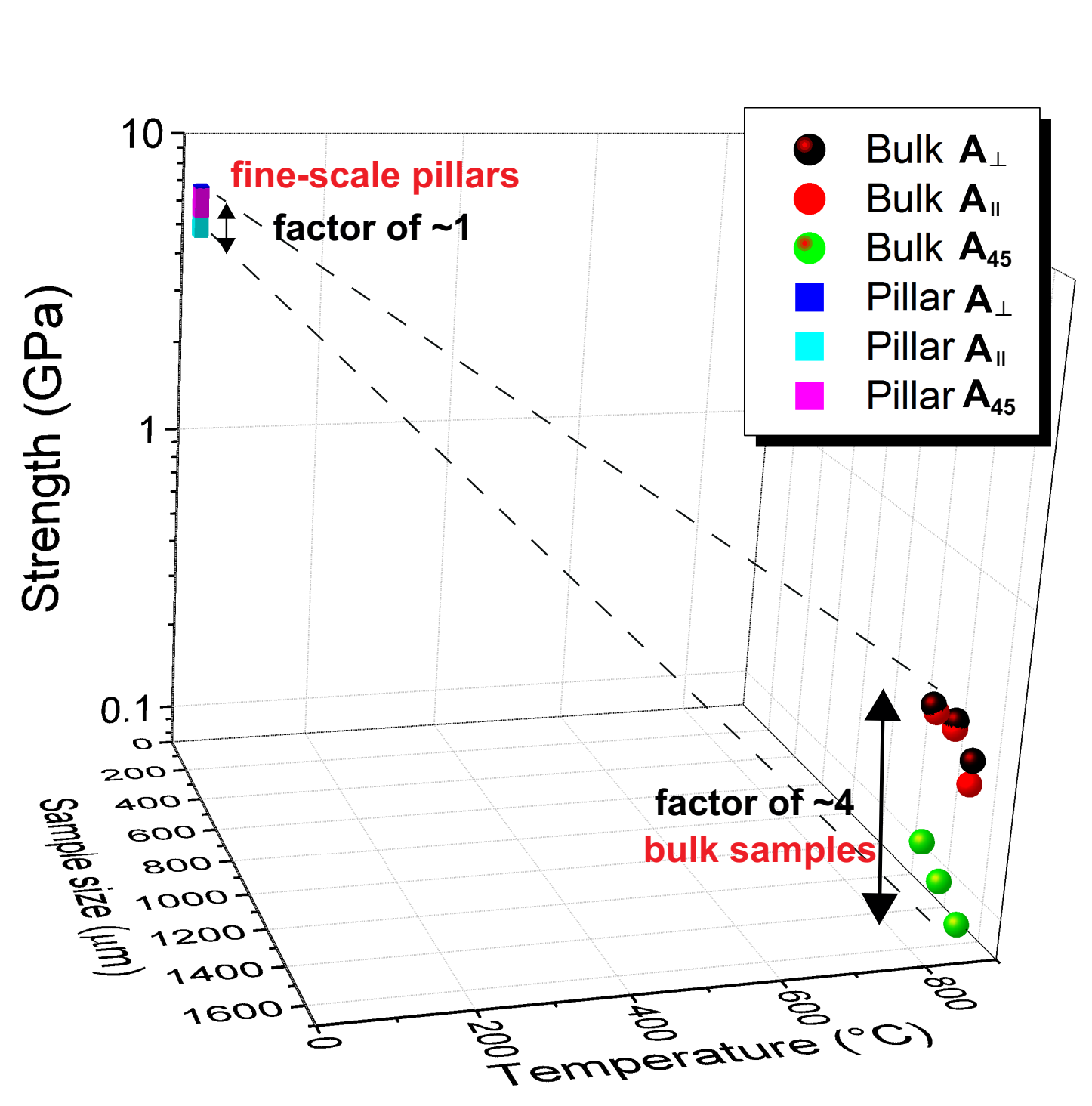

Figure 6 
Figure 7

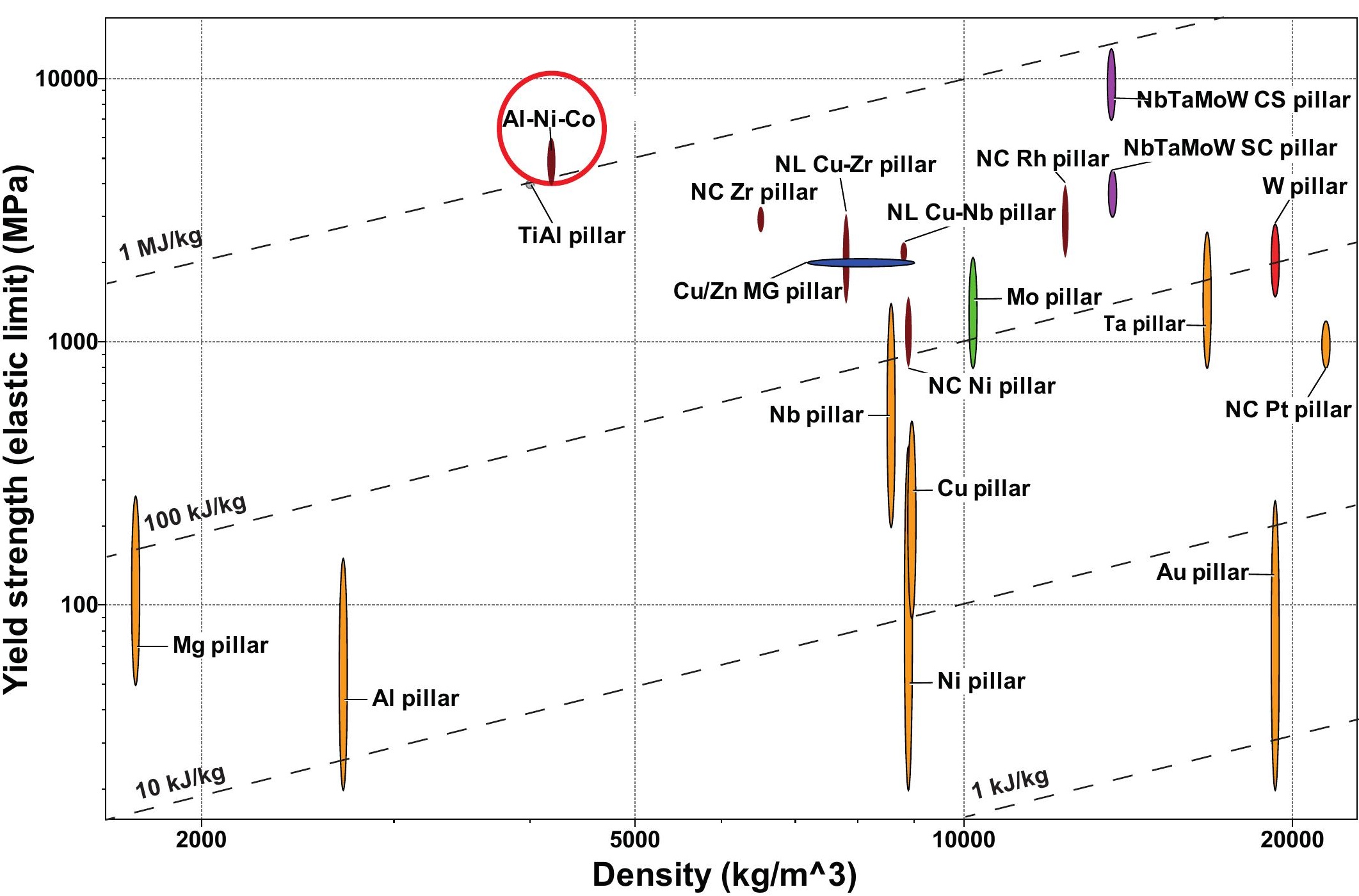

